\title{
Controllability for the Impulsive Semilinear Nonlocal Fuzzy Integrodifferential Equations in n-Dimensional Fuzzy Vector Space
}

\author{
Young Chel Kwun, ${ }^{1}$ Jeong Soon Kim, ${ }^{1}$ Min Ji Park, ${ }^{1}$ \\ and Jin Han Park ${ }^{2}$ \\ ${ }^{1}$ Department of Mathematics, Dong-A University, Busan 604-714, Republic of Korea \\ ${ }^{2}$ Department of Applied Mathematics, Pukyong National University, Busan 608-737, Republic of Korea
}

Correspondence should be addressed to Jin Han Park, jihpark@pknu.ac.kr

Received 14 March 2010; Accepted 21 June 2010

Academic Editor: T. Bhaskar

Copyright (c) 2010 Young Chel Kwun et al. This is an open access article distributed under the Creative Commons Attribution License, which permits unrestricted use, distribution, and reproduction in any medium, provided the original work is properly cited.

We study the existence and uniqueness of solutions and nonlocal controllability for the impulsive semilinear nonlocal fuzzy integrodifferential equations in $n$-dimensional fuzzy vector space $\left(E_{N}\right)^{n}$ by using short-term perturbations techniques and Banach fixed point theorem. This is an extension of the result of Kwun et al. (Kwun et al., 2009) to impulsive system.

\section{Introduction}

The theory of differential equations with discontinuous trajectories during the last twenty years has been to a great extent stimulated by their numerous applications to problem arising in mechanics, electrical engineering, the theory of automatic control, medicine and biology. For the monographs of the theory of impulsive differential equations, see the papers of Bainov and Simenov [1], Lakshmikantham et al. [2] and Samoileuko and Perestyuk [3], where numerous properties of their solutions are studied and detailed bibliographies are given. Rogovchenko [4] followed the ideas of the theory of impulsive differential equations which treats the changes of the state of the evolution process due to a short-term perturbations whose duration can be negligible in comparison with the duration of the process as an instant impulses. In 2001, Lakshmikantham and McRae [5] studied basic results for fuzzy impulsive differential equations. Park et al. [6] studied the existence and uniqueness of fuzzy solutions and controllability for the impulsive semilinear fuzzy integrodifferential equations in onedimensional fuzzy vector space $E_{N}^{1}$. Rodríguez-López [7] studied periodic boundary value 
problems for impulsive fuzzy differential equations. Fuzzy integrodifferential equations are a field of interest, due to their applicability to the analysis of phenomena with memory where imprecision is inherent. Balasubramaniam and Muralisankar [8] proved the existence and uniqueness of fuzzy solutions for the semilinear fuzzy integrodifferential equation with nonlocal initial condition. They considered the semilinear one-dimensional heat equation on a connected domain $(0,1)$ for material with memory. In one-dimensional fuzzy vector space $E_{N}^{1}$, Park et al. [9] proved the existence and uniqueness of fuzzy solutions and presented the sufficient condition of nonlocal controllability for the following semilinear fuzzy integrodifferential equation with nonlocal initial condition.

In [10], Kwun et al. proved the existence and uniqueness of fuzzy solutions for the semilinear fuzzy integrodifferential equations by using successive iteration. In [11], Kwun et al. investigated the continuously initial observability for the semilinear fuzzy integrodifferential equations. Bede and Gal [12] studied almost periodic fuzzy-numbervalued functions. Gal and N'Guerekata [13] studied almost automorphic fuzzy-numbervalued functions. More recently, Kwun et al. [14] studied the existence and uniqueness of solutions and nonlocal controllability for the semilinear fuzzy integrodifferential equations in $n$-dimensional fuzzy vector space.

In this paper, we study the existence and uniqueness of solutions and nonlocal controllability for the following impulsive semilinear nonlocal fuzzy integrodifferential equations in $n$-dimensional fuzzy vector space by using short-term perturbations techniques and Banach fixed point theorem:

$$
\begin{gathered}
\frac{d x_{i}(t)}{d t}=A_{i}\left[x_{i}(t)+\int_{0}^{t} G(t-s) x_{i}(s) d s\right]+f_{i}\left(t, x_{i}(t), \int_{0}^{t} q_{i}\left(t, s, x_{i}(s)\right) d s\right)+u_{i}(t) \quad \text { on } E_{N^{\prime}}^{i} \\
x_{i}(0)+g_{i}\left(x_{i}\right)=x_{0_{i}} \in E_{N^{\prime}}^{i} \\
\Delta x_{i}\left(t_{k}\right)=I_{k}\left(x_{i}\left(t_{k}\right)\right), \quad t \neq t_{k}, k=1,2, \ldots, m, i=1,2, \ldots, n,
\end{gathered}
$$

where $A_{i}:[0, T] \rightarrow E_{N}^{i}$ is fuzzy coefficient, $E_{N}^{i}$ is the set of all upper semicontinuously convex fuzzy numbers on $R$ with $E_{N}^{i} \neq E_{N}^{j}(i \neq j), f_{i}:[0, T] \times E_{N}^{i} \times E_{N}^{i} \rightarrow E_{N}^{i}$ and $q_{i}:[0, T] \times$ $[0, T] \times E_{N}^{i}$ are nonlinear regular fuzzy functions, $g_{i}: E_{N}^{i} \rightarrow E_{N}^{i}$ is a nonlinear continuous function, $G(t)$ is an $n \times n$ continuous matrix such that $d G(t) x_{i} / d t$ is continuous for $x_{i} \in E_{N}^{i}$ and $t \in[0, T]$ with $\|G(t)\| \leq k, k>0, u_{i}:[0, T] \rightarrow E_{N}^{i}$ is a control function, $x_{0_{i}} \in E_{N}^{i}$ is an initial value and $I_{k} \in C\left(E_{N}^{i}, E_{N}^{i}\right)$ are bounded functions, $\Delta x_{i}\left(t_{k}\right)=x_{i}\left(t_{k}^{+}\right)-x_{i}\left(t_{k}^{-}\right)$, where $x_{i}\left(t_{k}^{-}\right)$ and $x_{i}\left(t_{k}^{+}\right)$represent the left and right limits of $x_{i}(t)$ at $t=t_{k}$, respectively.

\section{Preliminaries}

A fuzzy set $u$ of $R^{n}$ is a function $u: R^{n} \rightarrow[0,1]$. For each fuzzy set $u$, we denote by $[u]^{\alpha}=$ $\left\{x \in R^{n}: u(x) \geq \alpha\right\}$ for any $\alpha \in[0,1]$ its $\alpha$-level set.

Let $u, v$ be fuzzy sets of $R^{n}$. It is well known that $[u]^{\alpha}=[v]^{\alpha}$ for each $\alpha \in[0,1]$ implies $u=v$. 
Let $E^{n}$ denote the collection of all fuzzy sets of $R^{n}$ that satisfies the following conditions:

(1) $u$ is normal, that is, there exists an $x_{0} \in R^{n}$ such that $u\left(x_{o}\right)=1$;

(2) $u$ is fuzzy convex, that is, $u(\lambda x+(1-\lambda) y) \geq \min \{u(x), u(y)\}$ for any $x, y \in R^{n}$, $0 \leq \lambda \leq 1$

(3) $u(x)$ is upper semicontinuous, that is, $u\left(x_{0}\right) \geq \varlimsup_{k \rightarrow \infty} u\left(x_{k}\right)$ for any $x_{k} \in R^{n}(k=$ $0,1,2, \ldots), x_{k} \rightarrow x_{0}$

(4) $[u]^{0}$ is compact.

We call $u \in E^{n}$ an $n$-dimension fuzzy number.

Wang et al. [15] defined $n$-dimensional fuzzy vector space and investigated its properties.

For any $u_{i} \in E, i=1,2, \ldots, n$, we call the ordered one-dimension fuzzy number class $u_{1}, u_{2}, \ldots, u_{n}$ (i.e., the Cartesian product of one-dimension fuzzy number $u_{1}, u_{2}, \ldots, u_{n}$ ) an $n$ dimension fuzzy vector, denote it as $\left(u_{1}, u_{2}, \ldots, u_{n}\right)$, and call the collection of all $n$-dimension fuzzy vectors (i.e., the Cartesian product $\overbrace{E \times E \times \cdots \times E}$ ) $n$-dimensional fuzzy vector space, and denote it as $(E)^{n}$.

Definition 2.1 (see [15]). If $u \in E^{n}$, and $[u]^{\alpha}$ is a hyperrectangle, that is, $[u]^{\alpha}$ can be represented by $\prod_{i=1}^{n}\left[u_{i l}^{\alpha}, u_{i r}^{\alpha}\right]$, that is, $\left[u_{1 l}^{\alpha}, u_{1 r}^{\alpha}\right] \times\left[u_{2 l}^{\alpha}, u_{2 r}^{\alpha}\right] \times \cdots \times\left[u_{n l}^{\alpha}, u_{n r}^{\alpha}\right]$ for every $\alpha \in[0,1]$, where $u_{i l}^{\alpha}, u_{i r}^{\alpha} \in R$ with $u_{i l}^{\alpha} \leq u_{i r}^{\alpha}$ when $\alpha \in(0,1], i=1,2, \ldots, n$, then we call $u$ a fuzzy $n$-cell number. We denote the collection of all fuzzy $n$-cell numbers by $L\left(E^{n}\right)$.

Theorem 2.2 (see [15]). For any $u \in L\left(E^{n}\right)$ with $[u]^{\alpha}=\prod_{i=1}^{n}\left[u_{i l^{\prime}}^{\alpha} u_{i r}^{\alpha}\right](\alpha \in[0,1])$, there exists a unique $\left(u_{1}, u_{2}, \ldots, u_{n}\right) \in(E)^{n}$ such that $\left[u_{i}\right]^{\alpha}=\left[u_{i l}^{\alpha}, u_{i r}^{\alpha}\right](i=1,2, \ldots, n$ and $\alpha \in[0,1])$. Conversely, for any $\left(u_{1}, u_{2}, \ldots, u_{n}\right) \in(E)^{n}$ with $\left[u_{i}\right]^{\alpha}=\left[u_{i l}^{\alpha}, u_{i r}^{\alpha}\right](i=1,2, \ldots, n$ and $\alpha \in[0,1])$, there exists a unique $u \in L\left(E^{n}\right)$ such that $[u]^{\alpha}=\prod_{i=1}^{n}\left[u_{i l}^{\alpha}, u_{i r}^{\alpha}\right](\alpha \in[0,1])$.

Note 1 (see [15]). Theorem 2.2 indicates that fuzzy $n$-cell numbers and $n$-dimension fuzzy vectors can represent each other, so $L\left(E^{n}\right)$ and $(E)^{n}$ may be regarded as identity. If $\left(u_{1}, u_{2}, \ldots, u_{n}\right) \in(E)^{n}$ is the unique $n$-dimension fuzzy vector determined by $u \in L\left(E^{n}\right)$, then we denote $u=\left(u_{1}, u_{2}, \ldots, u_{n}\right)$.

Let $\left(E_{N}^{i}\right)^{n}=E_{N}^{1} \times E_{N}^{2} \times \cdots \times E_{N}^{n}$, where $E_{N}^{i}(i=1,2, \ldots, n)$ is a fuzzy subset of $R$. Then $\left(E_{N}^{i}\right)^{n} \subseteq(E)^{n}$.

Definition 2.3 (see [15]). The complete metric $D_{L}$ on $\left(E_{N}^{i}\right)^{n}$ is defined by

$$
D_{L}(u, v)=\sup _{0<\alpha \leq 1} d_{L}\left([u]^{\alpha},[v]^{\alpha}\right)=\sup _{0<\alpha \leq 1 \leq i \leq n}\left\{\left|u_{i l}^{\alpha}-v_{i l}^{\alpha}\right|,\left|u_{i r}^{\alpha}-v_{i r}^{\alpha}\right|\right\}
$$

for any $u, v \in\left(E_{N}^{i}\right)^{n}$, which satisfies $d_{L}(u+w, v+w)=d_{L}(u, v)$.

Definition 2.4. Let $u, v \in C\left([0, T]:\left(E_{N}^{i}\right)^{n}\right)$,

$$
H_{1}(u, v)=\sup _{0 \leq t \leq T} D_{L}(u(t), v(t))
$$


Definition 2.5 (see [15]). The derivative $x^{\prime}(t)$ of a fuzzy process $x \in\left(E_{N}^{i}\right)^{n}$ is defined by

$$
\left[x^{\prime}(t)\right]^{\alpha}=\prod_{i=1}^{n}\left[\left(x_{i l}^{\alpha}\right)^{\prime}(t),\left(x_{i r}^{\alpha}\right)^{\prime}(t)\right]
$$

provided that equation defines a fuzzy $x^{\prime}(t) \in\left(E_{N}^{i}\right)^{n}$.

Definition 2.6 (see [15]). The fuzzy integral $\int_{b}^{a} x(t) d t, a, b \in[0, T]$ is defined by

$$
\left[\int_{b}^{a} x(t) d t\right]^{\alpha}=\prod_{i=1}^{n}\left[\int_{b}^{a} x_{i l}^{\alpha}(t) d t \int_{b}^{a} x_{i r}^{\alpha}(t) d t\right]
$$

provided that the Lebesgue integrals on the right-hand side exist.

\section{Existence and Uniqueness}

In this section we consider the existence and uniqueness of the fuzzy solution for $(1.1)(u \equiv 0)$. We define

$$
\begin{gathered}
A=\left(A_{1}, A_{2}, \ldots, A_{n}\right), \\
x=\left(x_{1}, x_{2}, \ldots, x_{n}\right), \\
f=\left(f_{1}, f_{2}, \ldots, f_{n}\right), \\
q=\left(q_{1}, q_{2}, \ldots, q_{n}\right), \\
u=\left(u_{1}, u_{2}, \ldots, u_{n}\right), \\
g=\left(g_{1}, g_{2}, \ldots, g_{n}\right), \\
x_{0}=\left(x_{0_{1}}, x_{0_{2}}, \ldots, x_{0_{n}}\right) .
\end{gathered}
$$

Then

$$
A, x, f, q, x_{0}, u, g \in\left(E_{N}^{i}\right)^{n}
$$

Instead of (1.1), we consider the following fuzzy integrodifferential equations in $\left(E_{N}^{i}\right)^{n}$

$$
\begin{gathered}
\frac{d x(t)}{d t}=A\left[x(t)+\int_{0}^{t} G(t-s) x(s) d s\right]+f\left(t, x(t), \int_{0}^{t} q(t, s, x(s)) d s\right)+u(t) \text { on }\left(E_{N}^{i}\right)^{n}, \\
x(0)+g(x)=x_{0} \in\left(E_{N}^{i}\right)^{n}, \\
\Delta x\left(t_{k}\right)=I_{k}\left(x\left(t_{k}\right)\right), \quad t \neq t_{k}, k=1,2, \ldots, m, i=1,2, \ldots, n
\end{gathered}
$$


with fuzzy coefficient $A:[0, T] \rightarrow\left(E_{N}^{i}\right)^{n}$, initial value $x_{0} \in\left(E_{N}^{i}\right)^{n}$, and $u:[0, T] \rightarrow\left(E_{N}^{i}\right)^{n}$ being a control function. Given nonlinear regular fuzzy functions $f:[0, T] \times\left(E_{N}^{i}\right)^{n} \times\left(E_{N}^{i}\right)^{n} \rightarrow$ $\left(E_{N}^{i}\right)^{n}$ and $q:[0, T] \times[0, T] \times\left(E_{N}^{i}\right)^{n} \rightarrow\left(E_{N}^{i}\right)^{n}$ satisfy global Lipschitz conditions, that is, there exist finite constants $k_{1}, k_{2}, M>0$ such that

$$
\begin{aligned}
& d_{L}\left(\left[f\left(s, \xi_{1}(s), \eta_{1}(s)\right)\right]^{\alpha},\left[f\left(s, \xi_{2}(s), \eta_{2}(s)\right)\right]^{\alpha}\right) \\
& \quad \leq k_{1} d_{L}\left(\left[\xi_{1}(s)\right]^{\alpha},\left[\xi_{2}(s)\right]^{\alpha}\right)+k_{2} d_{L}\left(\left[\eta_{1}(s)\right]^{\alpha},\left[\eta_{2}(s)\right]^{\alpha}\right), \\
& d_{L}\left(\left[q\left(t, s, \varphi_{1}(s)\right)\right]^{\alpha},\left[q\left(t, s, \varphi_{2}(s)\right)\right]^{\alpha}\right) \leq M d_{L}\left(\left[\varphi_{1}(s)\right]^{\alpha},\left[\varphi_{2}(s)\right]^{\alpha}\right)
\end{aligned}
$$

for all $\xi_{j}(s), \eta_{j}(s), \varphi_{j}(s) \in\left(E_{N}^{i}\right)^{n}(j=1,2)$, the nonlinear function $g:\left(E_{N}^{i}\right)^{n} \rightarrow\left(E_{N}^{i}\right)^{n}$ is continuous and satisfies the Lipschitz condition

$$
d_{L}\left([g(x(\cdot))]^{\alpha},[g(y(\cdot))]^{\alpha}\right) \leq h d_{L}\left([x(\cdot)]^{\alpha},[y(\cdot)]^{\alpha}\right)
$$

for all $x(\cdot), y(\cdot) \in\left(E_{N}^{i}\right)^{n}, h$ is a finite positive constant.

Definition 3.1. The fuzzy process $x: I=[0, T] \rightarrow\left(E_{N}^{i}\right)^{n}$ with $\alpha$-level set $[x(t)]^{\alpha}=\Pi_{i=1}^{n}\left[x_{i}\right]^{\alpha}=$ $\prod_{i=1}^{n}\left[x_{i l}^{\alpha}, x_{i r}^{\alpha}\right]$ is a fuzzy solution of (3.4) and (3.5) without nonhomogeneous term if and only if

$$
\begin{aligned}
& \left(x_{i l}^{\alpha}\right)^{\prime}(t)=\min \left\{A_{i j}^{\alpha}(t)\left[x_{i k}^{\alpha}(t)+\int_{0}^{t} G(t-s) x_{i k}^{\alpha}(s) d s\right]: j, k=l, r\right\}, \\
& \left(x_{i r}^{\alpha}\right)^{\prime}(t)=\max \left\{A_{i j}^{\alpha}(t)\left[x_{i k}^{\alpha}(t)+\int_{0}^{t} G(t-s) x_{i k}^{\alpha}(s) d s\right]: j, k=l, r\right\}, \\
& x_{i l}^{\alpha}(0)+g_{i l}^{\alpha}\left(x_{i l}^{\alpha}\right)=x_{0_{i l} l^{\prime}}^{\alpha} \quad x_{i r}^{\alpha}(0)+g_{i r}^{\alpha}\left(x_{i r}^{\alpha}\right)=x_{0_{i r}}^{\alpha}, \quad i=1,2, \ldots, n .
\end{aligned}
$$

For the sequel, we need the following assumption:

(H1) $S(t)$ is a fuzzy number satisfying, for $y \in\left(E_{N}^{i}\right)^{n},(d / d t) S(t) y \in C^{1}(I$ : $\left.\left(E_{N}^{i}\right)^{n}\right) \cap C\left(I:\left(E_{N}^{i}\right)^{n}\right)$, the equation

$$
\frac{d}{d t} S(t) y=A\left[S(t) y+\int_{0}^{t} G(t-s) S(s) y d s\right], \quad t \in I,
$$

where

$$
[S(t)]^{\alpha}=\prod_{i=1}^{n}\left[S_{i}(t)\right]^{\alpha}=\prod_{i=1}^{n}\left[S_{i l}^{\alpha}(t), S_{i r}^{\alpha}(t)\right], \quad S(0)=I
$$

and $S_{i j}^{\alpha}(t)(j=l, r)$ is continuous with $\left|S_{i j}^{\alpha}(t)\right| \leq c, c>0$, for all $t \in I=[0, T]$. 
In order to define the solution of (3.4)-(3.6), we will consider the space $\Omega_{i}=\left\{x_{i}: J \rightarrow\right.$ $E_{N}^{i}:\left(x_{i}\right)_{k} \in C\left(J_{k}, E_{N}^{i}\right), J_{k}=\left(t_{k}, t_{k+1}\right], k=0,1, \ldots, m$, and there exist $\left(x_{i}\right)\left(t_{k}^{-}\right)$and $\left(x_{i}\right)\left(t_{k}^{+}\right)(k=$ $1,2, \ldots, m)$, with $\left.\left(x_{i}\right)\left(t_{k}^{-}\right)=\left(x_{i}\right)\left(t_{k}\right)\right\}, i=1,2, \ldots, n$.

Let $\Omega^{\prime}=\prod_{i=1}^{n} \Omega_{i}^{\prime}, \Omega_{i}^{\prime}=\Omega_{i} \cap C\left([0, T]: E_{N}^{i}\right), i=1,2, \ldots, n$.

Lemma 3.2. If $x$ is an integral solution of (3.4)-(3.6) $(u \equiv 0)$, then $x$ is given by

$$
\begin{aligned}
x(t)= & S(t)\left(x_{0}-g(x)\right)+\int_{0}^{t} S(t-s) f\left(s, x(s), \int_{0}^{s} q(s, \tau, x(\tau)) d \tau\right) d s \\
& +\sum_{0<t_{k}<t} S\left(t-t_{k}\right) I_{k}\left(x\left(t_{k}^{-}\right)\right), \quad \text { for } t \in J .
\end{aligned}
$$

Proof. Let $x$ be a solution of (3.4)-(3.6). Define $\omega(s)=S(t-s) x(s)$. Then we have that

$$
\begin{aligned}
\frac{d \omega(s)}{d s} & =-\frac{d S(t-s)}{d s} x(s)+S(t-s) \frac{d x(s)}{d s} \\
& =-A\left[S(t-s) x(s)+\int_{0}^{t} G(t-s) S(s) x(s) d s\right]+S(t-s) \frac{d x(s)}{d s} \\
& =S(t-s) f\left(s, x(s), \int_{0}^{s} q(s, \tau, x(\tau)) d \tau\right) .
\end{aligned}
$$

Consider $t_{k}<t, k=1,2, \ldots, m$. Then integrating the previous equation, we have

$$
\int_{0}^{t} \frac{d \omega(s)}{d s} d s=\int_{0}^{t} S(t-s) f\left(s, x(s), \int_{0}^{s} q(s, \tau, x(\tau)) d \tau\right) d s
$$

For $k=1$,

$$
\omega(t)-\omega(0)=\int_{0}^{t} S(t-s) f\left(s, x(s), \int_{0}^{s} q(s, \tau, x(\tau)) d \tau\right) d s
$$

or

$$
x(t)=S(t)\left(x_{0}-g(x)\right)+\int_{0}^{t} S(t-s) f\left(s, x(s), \int_{0}^{s} q(s, \tau, x(\tau)) d \tau\right) d s .
$$


Now for $k=2, \ldots, m$, we have that

$$
\begin{gathered}
\int_{0}^{t_{1}} \frac{d \omega(s)}{d s} d s+\int_{t_{1}}^{t_{2}} \frac{d \omega(s)}{d s} d s+\cdots+\int_{t_{k}}^{t} \frac{d \omega(s)}{d s} d s \\
=\int_{0}^{t} S(t-s) f\left(s, x(s), \int_{0}^{s} q(s, \tau, x(\tau)) d \tau\right) d s .
\end{gathered}
$$

Then

$$
\begin{array}{r}
\omega\left(t_{1}^{-}\right)-\omega(0)+\omega\left(t_{2}^{-}\right)-\omega\left(t_{1}^{+}\right)+\cdots-\omega\left(t_{k}^{+}\right)+\omega(t) \\
=\int_{0}^{t} S(t-s) f\left(s, x(s), \int_{0}^{s} q(s, \tau, x(\tau)) d \tau\right) d s
\end{array}
$$

if and only if

$$
\omega(t)=\omega(0)+\int_{0}^{t} S(t-s) f\left(s, x(s), \int_{0}^{s} q(s, \tau, x(\tau)) d \tau\right) d s+\sum_{0<t_{k}<t}\left[\omega\left(t_{k}^{+}\right)-\omega\left(t_{k}^{-}\right)\right]
$$

Hence

$$
x(t)=S(t)\left(x_{0}-g(x)\right)+\int_{0}^{t} S(t-s) f\left(s, x(s), \int_{0}^{s} q(s, \tau, x(\tau)) d \tau\right) d s+\sum_{0<t_{k}<t} S\left(t-t_{k}\right) I_{k}\left(x\left(t_{k}^{-}\right)\right),
$$

which proves the lemma.

Assume the following:

(H2) there exists $d>0$ such that

$$
d_{L}\left(\left[I_{k}\left(x\left(t_{k}^{-}\right)\right)\right]^{\alpha},\left[I_{k}\left(y\left(t_{k}^{-}\right)\right)\right]^{\alpha}\right) \leq d d_{L}\left([x(t)]^{\alpha},[y(t)]^{\alpha}\right),
$$

where $x(t), y(t) \in \Omega^{\prime} ;$

(H3)

$$
c\left[T\left\{h(1+c)+c d+k_{1}\left(1+\frac{c T}{2}\right)+k_{2} M T\left(12+\frac{c T}{3}\right)\right\}+(h+d)\right]<1 .
$$


Theorem 3.3. Let $T>0$. If hypotheses (H1)-(H3) are hold, then, for every $x_{0} \in\left(E_{N}^{i}\right)^{n},(3.13)$ has a unique fuzzy solution $x \in \Omega^{\prime}$.

Proof. For each $x(t) \in \Omega^{\prime}$ and $t \in[0, T]$, define $\left(G_{0} x\right)(t) \in \Omega^{\prime}$ by

$$
\begin{aligned}
\left(G_{0} x\right)(t)= & S(t)\left(x_{0}-g(x)\right)+\int_{0}^{t} S(t-s) f\left(s, x(s), \int_{0}^{s} q(s, \tau, x(\tau)) d \tau\right) d s \\
& +\sum_{0<t_{k}<t} S\left(t-t_{k}\right) I_{k}\left(x\left(t_{k}^{-}\right)\right) .
\end{aligned}
$$

Thus, $G_{0} x:[0, T] \rightarrow \Omega^{\prime}$ is continuous, so $G_{0}$ is a mapping from $\Omega^{\prime}$ into itself. By Definitions 2.3 and 2.4, some properties of $d_{L}$ and inequalities (3.7), (3.8), and (3.9), we have the following inequalities. For $x, y \in \Omega^{\prime}$,

$$
\begin{aligned}
d_{L}\left(\left[\left(G_{0} x\right)(t)\right]^{\alpha},\left[\left(G_{0} y\right)(t)\right]^{\alpha}\right) & \\
\leq & d_{L}\left(\left[S(t)\left(x_{0}-g(x)\right)+\int_{0}^{t} S(t-s) f\left(s, x(s), \int_{0}^{s} q(s, \tau, x(\tau)) d \tau\right) d s\right]^{\alpha}\right. \\
& {\left.\left[S(t)\left(x_{0}-g(y)\right)+\int_{0}^{t} S(t-s) f\left(s, y(s), \int_{0}^{s} q(s, \tau, y(\tau)) d \tau\right) d s\right]^{\alpha}\right) } \\
+ & d_{L}\left(\left[\sum_{0<t_{k}<t} S\left(t-t_{k}\right) I_{k}\left(x\left(t_{k}^{-}\right)\right)\right]^{\alpha},\left[\sum_{0<t_{k}<t} S\left(t-t_{k}\right) I_{k}\left(y\left(t_{k}^{-}\right)\right)\right]^{\alpha}\right) \\
\leq & d_{L}\left([S(t) g(x)]^{\alpha},[S(t) g(y)]^{\alpha}\right) \\
+ & \int_{0}^{t} d_{L}\left(\left[S(t-s) f\left(s, x(s), \int_{0}^{s} q(s, \tau, x(\tau)) d \tau\right)\right]^{\alpha},\right. \\
& +d_{L}\left(\left[\sum_{0<t_{k}<t} S\left(t-t_{k}\right) I_{k}\left(x\left(t_{k}^{-}\right)\right)\right]^{\alpha},\left[\sum_{0<t_{k}<t} S\left(t-t_{k}\right) I_{k}\left(y\left(t_{k}^{-}\right)\right)\right]^{\alpha}\right) \\
\leq & c h d_{L}\left([x(\cdot)]^{\alpha},[y(\cdot)]^{\alpha}\right) \\
+ & +c \int_{0}^{t}\left(k_{1} d_{L}\left([x(s)]^{\alpha},[y(s)]^{\alpha}\right)+k_{2} M \int_{0}^{s} d_{L}\left([x(\tau)]^{\alpha},[y(\tau)]^{\alpha}\right) d \tau\right) d s \\
+ & c d d_{L}\left([x(t)]^{\alpha},[y(t)]^{\alpha}\right) . \\
&
\end{aligned}
$$


Therefore

$$
\begin{aligned}
& D_{L}\left(\left(G_{0} x\right)(t),\left(G_{0} y\right)(t)\right) \\
& =\sup _{0<\alpha \leq 1} d_{L}\left(\left[\left(G_{0} x\right)(t)\right]^{\alpha},\left[\left(G_{0} y\right)(t)\right]^{\alpha}\right) \\
& \leq \quad c h \sup _{0<\alpha \leq 1} d_{L}\left([x(\cdot)]^{\alpha},[y(\cdot)]^{\alpha}\right) \\
& \quad+c \int_{0}^{t}\left(k_{1} \sup _{0<\alpha \leq 1} d_{L}\left([x(s)]^{\alpha},[y(s)]^{\alpha}\right)+k_{2} M \int_{0}^{s} \sup _{0<\alpha \leq 1} d_{L}\left([x(\tau)]^{\alpha},[y(\tau)]^{\alpha}\right) d \tau\right) d s \\
& \quad+c d \sup _{0<\alpha \leq 1} d_{L}\left([x(t)]^{\alpha},[y(t)]^{\alpha}\right) \\
& \leq c h D_{L}(x(\cdot), y(\cdot)) \\
& \quad+c \int_{0}^{t}\left(k_{1} D_{L}(x(s), y(s))+k_{2} M \int_{0}^{s} D_{L}(x(\tau), y(\tau)) d \tau\right) d s+c d D_{L}(x(t), y(t)) .
\end{aligned}
$$

Hence

$$
\begin{aligned}
H_{1}\left(G_{0} x, G_{0} y\right)= & \sup _{0 \leq t \leq T} D_{L}\left(\left(G_{0} x\right)(t),\left(G_{0} y\right)(t)\right) \\
\leq & c \sup _{0 \leq t \leq T} D_{L}(x(\cdot), y(\cdot)) \\
& +c \sup _{0 \leq t \leq T} \int_{0}^{t}\left(k_{1} D_{L}(x(s), y(s))+k_{2} M \int_{0}^{s} D_{L}(x(\tau), y(\tau)) d \tau\right) d s \\
& +c d \sup _{0 \leq t \leq T} D_{L}(x(t), y(t)) \\
\leq & c\left(h+d+\left(k_{1}+k_{2} M \frac{T}{2}\right) T\right) H_{1}(x, y) .
\end{aligned}
$$

By hypothesis (H3), $G_{0}$ is a contraction mapping. Using the Banach fixed point theorem, (3.13) has a unique fixed point $x \in \Omega^{\prime}$.

\section{Nonlocal Controllability}

In this section, we show the nonlocal controllability for the control system (1.1). 
The control system (1.1) is related to the following fuzzy integral system:

$$
\begin{aligned}
x(t)= & S(t)\left(x_{0}-g(x)\right)+\int_{0}^{t} S(t-s) f\left(s, x(s), \int_{0}^{s} q(s, \tau, x(\tau)) d \tau\right) d s \\
& +\int_{0}^{t} S(t-s) u(s) d s+\sum_{0<t_{k}<t} S\left(t-t_{k}\right) I_{k}\left(x\left(t_{k}^{-}\right)\right) .
\end{aligned}
$$

Definition 4.1. Equations (1.1)-(3) are nonlocal controllable. Then there exists $u(t)$ such that the fuzzy solution $x(t)$ for $(4.1)$ as $x(T)=x^{1}-g(x)$ (i.e., $\left.[x(T)]^{\alpha}=\left[x^{1}-g(x)\right]^{\alpha}\right)$, where $x^{1} \in$ $\left(E_{N}^{i}\right)^{n}$, is target set.

Define the fuzzy mapping $\tilde{\beta}: \tilde{P}\left(R^{n}\right) \rightarrow\left(E_{N}^{i}\right)^{n}$ by

$$
\tilde{\beta}^{\alpha}(v)= \begin{cases}\int_{0}^{T} S^{\alpha}(T-s) v(s) d s, & v \subset \bar{\Gamma}_{u} \\ 0, & \text { otherwise }\end{cases}
$$

where $\bar{\Gamma}_{u}$ is closed support of $u$. Then there exists

$$
\tilde{\beta}_{i}: \tilde{P}(R) \longrightarrow E_{N}^{i} \quad(i=1,2, \ldots, n)
$$

such that

$$
\tilde{\beta}_{i}^{\alpha}\left(v_{i}\right)= \begin{cases}\int_{0}^{T} S_{i}^{\alpha}(T-s) v_{i}(s) d s, & v_{i}(s) \subset \bar{\Gamma}_{u_{i}} \\ 0, & \text { otherwise }\end{cases}
$$

Then there exists $\tilde{\beta}_{i j}^{\alpha}(j=l, r)$ such that

$$
\begin{aligned}
& \tilde{\beta}_{i l}^{\alpha}\left(v_{i l}\right)=\int_{0}^{T} S_{i l}^{\alpha}(T-s) v_{i l}(s) d s, \quad v_{i l}(s) \in\left[u_{i l}^{\alpha}(s), u_{i}^{1}\right] \\
& \tilde{\beta}_{i r}^{\alpha}\left(v_{i r}\right)=\int_{0}^{T} S_{i r}^{\alpha}(T-s) v_{i r}(s) d s, \quad v_{i r}(s) \in\left[u_{i}^{1}, u_{i r}^{\alpha}(s)\right] .
\end{aligned}
$$

We assume that $\tilde{\beta}_{i l}^{\alpha}, \tilde{\beta}_{i r}^{\alpha}$ are bijective mappings. 
We can introduce $\alpha$-level set of $u(s)$ of (4.1):

$$
\begin{aligned}
{[u(s)]^{\alpha}=\prod_{i=1}^{n}\left[u_{i}(s)\right]^{\alpha}=} & \prod_{i=1}^{n}\left[u_{i l}^{\alpha}(s), u_{i r}^{\alpha}(s)\right] \\
=\prod_{i=1}^{n}\left[\left(\tilde{\beta}_{i l}^{\alpha}\right)^{-1}(\right. & \left(\left(x^{1}\right)_{i l}^{\alpha}-g_{i l}^{\alpha}\left(x_{i l}^{\alpha}\right)\right)-S_{i l}^{\alpha}(T)\left(x_{0_{i l}}^{\alpha}-g_{i l}^{\alpha}\left(x_{i l}^{\alpha}\right)\right) \\
& -\int_{0}^{T} S_{i l}^{\alpha}(T-s) f_{i l}^{\alpha}\left(s, x_{i l}^{\alpha}(s), \int_{0}^{s} q_{i l}^{\alpha}\left(s, \tau, x_{i l}^{\alpha}(\tau)\right) d \tau\right) d s \\
& \left.-\sum_{0<t_{k}<T} S_{i l}^{\alpha}\left(T-t_{k}\right) I_{k_{i l}}^{\alpha}\left(x_{i l}^{\alpha}\left(t_{k}^{-}\right)\right)\right), \\
\left(\tilde{\beta}_{i r}^{\alpha}\right)^{-1}( & \left(\left(x^{1}\right)_{i r}^{\alpha}-g_{i r}^{\alpha}\left(x_{i r}^{\alpha}\right)\right)-S_{i r}^{\alpha}(T)\left(x_{0_{i r}}^{\alpha}-g_{i r}^{\alpha}\left(x_{i r}^{\alpha}\right)\right) \\
& \left.-\int_{0}^{T} S_{i r}^{\alpha}(T-s) f_{i r}^{\alpha}\left(s, x_{i r}^{\alpha}(s), \int_{0}^{s} q_{i r}^{\alpha}\left(s, \tau, x_{i r}^{\alpha}(\tau)\right) d \tau\right)\right) d s \\
& \left.\left.-\sum_{0<t_{k}<T} S_{i r}^{\alpha}\left(T-t_{k}\right) I_{k_{i r}}^{\alpha}\left(x_{i r}^{\alpha}\left(t_{k}^{-}\right)\right)\right)\right] .
\end{aligned}
$$

Then substituting this expression into (4.1) yields $\alpha$-level of $x(T)$.

For each $i=1,2, \ldots, n$,

$$
\begin{aligned}
& {\left[x_{i}(T)\right]^{\alpha}=\left[S_{i l}^{\alpha}(T)\left(x_{0_{i l}}^{\alpha}-g_{i l}^{\alpha}\left(x_{i l}^{\alpha}\right)\right)+\int_{0}^{T} S_{i l}^{\alpha}(T-s)\right.} \\
& \quad \times f_{i l}^{\alpha}\left(s, x_{i l}^{\alpha}(s), \int_{0}^{s} q_{i l}^{\alpha}\left(s, \tau, x_{i l}^{\alpha}(\tau)\right) d \tau\right) d s+\sum_{0<t_{k}<T} S_{i l}^{\alpha}\left(T-t_{k}\right) I_{k_{i l}}^{\alpha}\left(x_{i l}^{\alpha}\left(t_{k}^{-}\right)\right) \\
& +\int_{0}^{T} S_{i l}^{\alpha}(T-s)\left(\tilde{\beta}_{i l}^{\alpha}\right)^{-1}\left(\left(\left(x^{1}\right)_{i l}^{\alpha}-g_{i l}^{\alpha}\left(x_{i l}^{\alpha}\right)\right)-S_{i l}^{\alpha}(T)\left(x_{0_{i l}}^{\alpha}-g_{i l}^{\alpha}\left(x_{i l}^{\alpha}\right)\right)\right. \\
& \quad-\int_{0}^{T} S_{i l}^{\alpha}(T-s) f_{i l}^{\alpha}\left(s, x_{i l}^{\alpha}(s), \int_{0}^{s} q_{i l}^{\alpha}\left(s, \tau, x_{i l}^{\alpha}(\tau)\right) d \tau\right) d s \\
& \left.\quad-\sum_{0<t_{k}<T} S_{i l}^{\alpha}\left(T-t_{k}\right) I_{k_{i l}}^{\alpha}\left(x_{i l}^{\alpha}\left(t_{k}^{-}\right)\right)\right) d s,
\end{aligned}
$$




$$
\begin{gathered}
S_{i r}^{\alpha}(T)\left(x_{0_{i r}}^{\alpha}-g_{i r}^{\alpha}\left(x_{i r}^{\alpha}\right)\right)+\int_{0}^{T} S_{i r}^{\alpha}(T-s) \times f_{i r}^{\alpha}\left(s, x_{i r}^{\alpha}(s), \int_{0}^{s} q_{i l}^{\alpha}\left(s, \tau, x_{i r}^{\alpha}(\tau)\right) d \tau\right) d s \\
+\sum_{0<t_{k}<T} S_{i r}^{\alpha}\left(T-t_{k}\right) I_{k_{i r}}^{\alpha}\left(x_{i r}^{\alpha}\left(t_{k}^{-}\right)\right) \\
+\int_{0}^{T} S_{i r}^{\alpha}(T-s)\left(\tilde{\beta}_{i r}^{\alpha}\right)^{-1} \\
\times\left(\left(\left(\left(x^{1}\right)_{i r}^{\alpha}-g_{i r}^{\alpha}\left(x_{i r}^{\alpha}\right)\right)\right)-S_{i r}^{\alpha}(T)\left(x_{0_{i r}}^{\alpha}-g_{i r}^{\alpha}\left(x_{i r}^{\alpha}\right)\right)\right. \\
-\int_{0}^{T} S_{i r}^{\alpha}(T-s) f_{i r}^{\alpha}\left(s, x_{i r}^{\alpha}(s), \int_{0}^{s} q_{i l}^{\alpha}\left(s, \tau, x_{i r}^{\alpha}(\tau)\right) d \tau\right) d s \\
\left.\left.-\sum_{0<t_{k}<T} S_{i r}^{\alpha}\left(T-t_{k}\right) I_{k_{i r}}^{\alpha}\left(x_{i r}^{\alpha}\left(t_{k}^{-}\right)\right)\right) d s\right] \\
=\left[\left(x^{1}-g(x)\right)_{i l^{\prime}}^{\alpha}\left(x^{1}-g(x)\right)_{i r}^{\alpha}\right]=\left[\left(x^{1}-g(x)\right)_{i}\right]^{\alpha} .
\end{gathered}
$$

Therefore

$$
[x(T)]^{\alpha}=\prod_{i=1}^{n}\left[x_{i}(T)\right]^{\alpha}=\prod_{i=1}^{n}\left[\left(x^{1}-g(x)\right)_{i}\right]^{\alpha}=\left[x^{1}-g(x)\right]^{\alpha} .
$$

We now set

$$
\begin{gathered}
\Phi x(t)=S(t)\left(x_{0}-g(x)\right)+\int_{0}^{t} S(t-s) f\left(s, x(s), \int_{0}^{t} q(s, \tau, x(\tau)) d \tau\right) d s \\
+\sum_{0<t_{k}<t} S\left(t-t_{k}\right) I_{k}\left(x\left(t_{k}^{-}\right)\right) \\
+\int_{0}^{t} S(t-s) \tilde{\beta}^{-1}\left(x^{1}-g(x)-S(T)\left(x_{0}-g(x)\right)\right. \\
-\int_{0}^{T} S(T-s) f\left(s, x(s), \int_{0}^{s} q(s, \tau, x(\tau)) d \tau\right) d s \\
\left.-\sum_{0<t_{k}<T} S\left(T-t_{k}\right) I_{k}\left(x\left(t_{k}^{-}\right)\right)\right) d s
\end{gathered}
$$

where the fuzzy mapping $\widetilde{\beta}^{-1}$ satisfies the previous statements.

Notice that $\Phi x(T)=x^{1}-g(x)$, which means that the control $u(t)$ steers (4.9) from the origin to $x^{1}-g(x)$ in time $T$ provided we can obtain a fixed point of the operator $\Phi$.

(H4) Assume that the linear system of $(4.9)(f \equiv 0)$ is controllable. 
Theorem 4.2. Suppose that hypotheses (H1)-(H4) are satisfied. Then (4.9) is nonlocal controllable.

Proof. We can easily check that $\Phi$ is continuous function from $\Omega^{\prime}$ to itself. By Definitions 2.3 and 2.4, some properties of $d_{L}$, and inequalities (3.7), (3.8), and (3.9), we have following inequalities. For any $x, y \in \Omega^{\prime}$,

$$
\begin{aligned}
& d_{L}\left([\Phi x(t)]^{\alpha},[\Phi y(t)]^{\alpha}\right) \\
& =d_{L}\left(\left[S(t)\left(x_{0}-g(x)\right)+\int_{0}^{t} S(t-s) f\left(s, x(s), \int_{0}^{s} q(s, \tau, x(\tau)) d \tau\right) d s\right.\right. \\
& +\sum_{0<t_{k}<t} S\left(t-t_{k}\right) I_{k}\left(x\left(t_{k}^{-}\right)\right) \\
& +\int_{0}^{t} S(t-s) \tilde{\beta}^{-1}\left(x^{1}-g(x)-S(T)\left(x_{0}-g(x)\right)\right. \\
& -\int_{0}^{T} S(T-s) f\left(s, x(s), \int_{0}^{s} q(s, \tau, x(\tau)) d \tau\right) d s \\
& \left.\left.-\sum_{0<t_{k}<T} S\left(T-t_{k}\right) I_{k}\left(x\left(t_{k}^{-}\right)\right)\right) d s\right]^{\alpha} \\
& {\left[S(t)\left(x_{0}-g(y)\right)+\int_{0}^{t} S(t-s) f\left(s, y(s), \int_{0}^{s} q(s, \tau, y(\tau)) d \tau\right) d s\right.} \\
& +\sum_{0<t_{k}<t} S\left(t-t_{k}\right) I_{k}\left(y\left(t_{k}^{-}\right)\right) \\
& +\int_{0}^{t} S(t-s) \tilde{\beta}^{-1}\left(x^{1}-g(y)-S(T)\left(x_{0}-g(y)\right)\right. \\
& -\int_{0}^{T} S(T-s) f\left(s, y(s), \int_{0}^{s} q(s, \tau, y(\tau)) d \tau\right) d s \\
& \left.\left.\left.-\sum_{0<t_{k}<T} S\left(T-t_{k}\right) I_{k}\left(y\left(t_{k}^{-}\right)\right)\right) d s\right]^{\alpha}\right) \\
& \leq \operatorname{chd}_{L}\left([x(\cdot)]^{\alpha},[y(\cdot)]^{\alpha}\right) \\
& +c \int_{0}^{t}\left(k_{1} d_{L}\left([x(s)]^{\alpha},[y(s)]^{\alpha}\right)+k_{2} M \int_{0}^{s} d_{L}\left([x(\tau)]^{\alpha},[y(\tau)]^{\alpha}\right) d \tau\right) d s \\
& +c d d_{L}\left([x(t)]^{\alpha},[y(t)]^{\alpha}\right) \\
& +c \int_{0}^{t}\left\{h d_{L}\left([x(\cdot)]^{\alpha},[y(\cdot)]^{\alpha}\right)+c h d_{L}\left([x(\cdot)]^{\alpha},[y(\cdot)]^{\alpha}\right)\right. \\
& +c \int_{0}^{T}\left(k_{1} d_{L}\left([x(s)]^{\alpha},[y(s)]^{\alpha}\right)+k_{2} M \int_{0}^{s} d_{L}\left([x(\tau)]^{\alpha},[y(\tau)]^{\alpha}\right) d \tau\right) d s \\
& \left.+c d d_{L}\left([x(t)]^{\alpha},[y(t)]^{\alpha}\right)\right\} d s
\end{aligned}
$$


Therefore

$$
\begin{aligned}
& D_{L}(\Phi x(t), \Phi y(t))=\sup _{0<\alpha \leq 1} d_{L}\left([\Phi x(t)]^{\alpha},[\Phi y(t)]^{\alpha}\right) \\
& \leq c h \sup _{0<\alpha \leq 1} d_{L}\left([x(\cdot)]^{\alpha},[y(\cdot)]^{\alpha}\right)+c d \sup _{0<\alpha \leq 1} d_{L}\left([x(t)]^{\alpha},[y(t)]^{\alpha}\right) \\
& +c \int_{0}^{t}\left(k_{1} \sup _{0<\alpha \leq 1} d_{L}\left([x(s)]^{\alpha},[y(s)]^{\alpha}\right)\right. \\
& \left.+k_{2} M \int_{0}^{s} \sup _{0<\alpha \leq 1} d_{L}\left([x(\tau)]^{\alpha},[y(\tau)]^{\alpha}\right) d \tau\right) d s \\
& +c \int_{0}^{t}\left\{h(1+c) \sup _{0<\alpha \leq 1} d_{L}\left([x(\cdot)]^{\alpha},[y(\cdot)]^{\alpha}\right)\right. \\
& +c \int_{0}^{T}\left(k_{1} \sup _{0<\alpha \leq 1} d_{L}\left([x(s)]^{\alpha},[y(s)]^{\alpha}\right)\right. \\
& \left.+k_{2} M \int_{0}^{s} \sup _{0<\alpha \leq 1} d_{L}\left([x(\tau)]^{\alpha},[y(\tau)]^{\alpha}\right) d \tau\right) d s \\
& \left.+c d \sup _{0<\alpha \leq 1} d_{L}\left([x(s)]^{\alpha},[y(s)]^{\alpha}\right)\right\} d s \\
& \leq c h D_{L}(x(\cdot), y(\cdot))+c d D_{L}(x(t), y(t)) \\
& +c \int_{0}^{t}\left(k_{1} D_{L}(x(s), y(s))+k_{2} M \int_{0}^{s} D_{L}(x(\tau), y(\tau)) d \tau\right) d s \\
& +c \int_{0}^{t}\left\{h(1+c) D_{L}(x(\cdot), y(\cdot))\right. \\
& +c \int_{0}^{T}\left(k_{1} D_{L}(x(s), y(s))+k_{2} M \int_{0}^{s} D_{L}(x(\tau), y(\tau)) d \tau\right) d s \\
& \left.+c d D_{L}(x(s), y(s))\right\} d s \text {. }
\end{aligned}
$$

Hence

$$
\begin{aligned}
H_{1}(\Phi x, \Phi y)= & \sup _{0 \leq t \leq T} D_{L}(\Phi x(t), \Phi y(t)) \\
\leq & c \sup _{0 \leq t \leq T} D_{L}(x(\cdot), y(\cdot))+c d \sup _{0 \leq t \leq T} D_{L}(x(t), y(t)) \\
& +c \sup _{0 \leq t \leq T} \int_{0}^{t}\left(k_{1} D_{L}(x(\cdot), y(\cdot))+k_{2} M \int_{0}^{s} D_{L}(x(\tau), y(\tau)) d \tau\right) d s
\end{aligned}
$$




$$
\begin{aligned}
& +c \sup _{0 \leq t \leq T} \int_{0}^{t}\left\{h(1+c) D_{L}(x(\cdot), y(\cdot))\right. \\
& +c \int_{0}^{T}\left(k_{1} D_{L}(x(s), y(s))+k_{2} M \int_{0}^{s} D_{L}(x(\tau), y(\tau)) d \tau\right) d s \\
& \left.+c d D_{L}(x(s), y(s))\right\} d s \\
& \leq c h H_{1}(x, y)+c d H_{1}(x, y)+c \int_{0}^{T}\left(k_{1} H_{1}(x, y)+k_{2} M \int_{0}^{s} H_{1}(x, y) d \tau\right) d s \\
& +c \int_{0}^{T}\left\{h(1+c) H_{1}(x, y)\right. \\
& \left.+c \int_{0}^{T}\left(k_{1} H_{1}(x, y)+k_{2} M \int_{0}^{s} H_{1}(x, y) d \tau\right) d s+c d H_{1}(x, y)\right\} d s \\
& =c\left[T\left\{h(1+c)+c d+k_{1}\left(1+\frac{c T}{2}\right)+k_{2} M T\left(\frac{1}{2}+\frac{c T}{3}\right)\right\}+(h+d)\right] H_{1}(x, y) \text {. }
\end{aligned}
$$

By hypothesis (H3), $\Phi$ is a contraction mapping. Using the Banach fixed point theorem, (4.9) has a unique fixed point $x \in \Omega^{\prime}$.

\section{Example}

Consider the two semilinear one-dimensional heat equations on a connected domain $(0,1)$ for material with memory on $E_{N}^{i}, i=1,2$, boundary condition

$$
x_{i}(t, 0)=x_{i}(t, 1)=0, \quad i=1,2
$$

and with initial conditions

$$
x_{i}\left(0, z_{i}\right)+\sum_{k=1}^{p}\left(c_{k}\right)_{i} x_{i}\left(t_{k}, z_{i}\right)=x_{0_{i}}\left(z_{i}\right)
$$

where $x_{0_{i}}\left(z_{i}\right) \in E_{N^{\prime}}^{i}$

$$
\sum_{k=1}^{p}\left(c_{k}\right)_{i} x_{i}\left(t_{k}, z_{i}\right)=g_{i}\left(x_{i}\right), \quad i=1,2
$$

Let $x_{i}\left(t, z_{i}\right), i=1,2$ be the internal energy and

$$
f_{i}\left(t, x_{i}\left(t, z_{i}\right)\right), \int_{0}^{t} q_{i}\left(t, s, x_{i}\left(s, z_{i}\right)\right) d s=\tilde{2} t x_{i}\left(t, z_{i}\right)^{2}+\int_{0}^{t}(t-s) x_{i}\left(s, z_{i}\right) d s, \quad i=1,2
$$


be the external heat with memory.

$$
\Delta x_{i}\left(t_{k}, z_{i}\right)=x_{i}\left(t_{k}^{+}, z_{i}\right)-x_{i}\left(t_{k}^{-}, z_{i}\right)
$$

is impulsive effect at $t=t_{k}(k=1,2, \ldots, m)$.

Let

$$
\begin{aligned}
& A=\left(A_{1}, A_{2}\right)=\left(\widetilde{2} \frac{\partial^{2}}{\partial z_{1}^{2}}, \widetilde{2} \frac{\partial^{2}}{\partial z_{2}^{2}}\right), \\
& f\left(t, x(t), \int_{0}^{t} q(t, s, x(s)) d s\right) \\
& =\left(f_{1}\left(t, x_{1}(t), \int_{0}^{t} q_{1}\left(t, s, x_{1}(s)\right) d s\right), f_{2}\left(t, x_{2}(t), \int_{0}^{t} q_{2}\left(t, s, x_{2}(s)\right) d s\right)\right) \\
& =\left(\tilde{2} t x_{1}\left(t, z_{1}\right)^{2}+\int_{0}^{t}(t-s) x_{1}\left(s, z_{1}\right) d s, \tilde{2} t x_{2}\left(t, z_{2}\right)^{2}+\int_{0}^{t}(t-s) x_{2}\left(s, z_{2}\right) d s\right), \\
& g(x)=\left(g_{1}\left(x_{1}\right), g_{2}\left(x_{2}\right)\right)=\left(\sum_{k=1}^{p}\left(c_{k}\right)_{1} x_{1}\left(t_{k}, z_{1}\right), \sum_{k=1}^{p}\left(c_{k}\right)_{2} x_{2}\left(t_{k}, z_{2}\right)\right), \\
& x(0)+g(x)=\left(x_{1}(0)+g_{1}(x), x_{2}(0)+g_{2}(x)\right), \quad x_{0}=\left(x_{0_{1}}, x_{0_{2}}\right)=(\widetilde{0}, \widetilde{0}), \\
& \Delta x\left(t_{k}\right)=\left(\Delta x_{1}\left(t_{k}\right), \Delta x_{2}\left(t_{k}\right)\right), \quad t \neq t_{k}, k=1,2, \ldots, m, \\
& I_{k}\left(x\left(t_{k}\right)\right)=\left(I_{k}\left(x_{1}\left(t_{k}\right)\right), I_{k}\left(x_{2}\left(t_{k}\right)\right)\right) \\
& =\left(x_{1}\left(t_{k}^{+}\right)-x_{1}\left(t_{k}^{-}\right), x_{2}\left(t_{k}^{+}\right)-x_{2}\left(t_{k}^{-}\right)\right) \\
& =\left(x_{1}\left(t_{k}^{+}, z_{1}\right)-x_{1}\left(t_{k}^{-}, z_{1}\right), x_{2}\left(t_{k}^{+}, z_{2}\right)-x_{2}\left(t_{k}^{-}, z_{2}\right)\right) \text {, } \\
& t \neq t_{k}, k=1,2, \ldots, m, \\
& G(t-s)=\left(e^{-(t-s)}, e^{-(t-s)}\right)
\end{aligned}
$$

then the balance equations become

$$
\begin{aligned}
\frac{d x(t)}{d t}=A & {\left[x(t)+\int_{0}^{t} G(t-s) x(s) d s\right] } \\
+ & f\left(t, x(t), \int_{0}^{t} q(t, s, x(s)) d s\right)+u(t) \text { on }\left(E_{N}^{i}\right)^{2}, \\
& x(0)+g(x)=x_{0} \in\left(E_{N}^{i}\right)^{2}, \\
\Delta x\left(t_{k}\right)= & I_{k}\left(x\left(t_{k}\right)\right), \quad t \neq t_{k}, k=1,2, \ldots, m, i=1,2 .
\end{aligned}
$$


The $\alpha$-level sets of fuzzy numbers are the following

$[\tilde{0}]^{\alpha}=[\alpha-1,1-\alpha],[\tilde{2}]^{\alpha}=[\alpha+1,3-\alpha]$ for all $\alpha \in[0,1]$. Then $\alpha$-level set of $f\left(t, x(t), \int_{0}^{t} q(t, s, x(s)) d s\right)$ is

$$
\begin{aligned}
{[f(t,} & \left.\left.x(t), \int_{0}^{t} q(t, s, x(s)) d s\right)\right]^{\alpha} \\
= & {\left[\tilde{2} t x_{1}(t)^{2}+\int_{0}^{t}(t-s) x_{1}(s) d s\right]^{\alpha} \times\left[\tilde{2} t x_{2}(t)^{2}+\int_{0}^{t}(t-s) x_{2}(s) d s\right]^{\alpha} } \\
= & \left([\tilde{2}]^{\alpha} \cdot t\left[x_{1}(t)^{2}\right]^{\alpha}+\int_{0}^{t}(t-s)\left[x_{1}(s)\right]^{\alpha} d s\right) \\
& \times\left([\tilde{2}]^{\alpha} \cdot t\left[x_{2}(t)^{2}\right]^{\alpha}+\int_{0}^{t}(t-s)\left[x_{2}(s)\right]^{\alpha} d s\right) \\
= & \left([\alpha+1,3-\alpha] \cdot t\left[\left(x_{1 l}^{\alpha}(t)\right)^{2},\left(x_{1 r}^{\alpha}(t)\right)^{2}\right]+\int_{0}^{t}(t-s)\left[\left(x_{1 l}^{\alpha}(s)\right),\left(x_{1 r}^{\alpha}(s)\right)\right] d s\right) \\
& \times\left([\alpha+1,3-\alpha] \cdot t\left[\left(x_{2 l}^{\alpha}(t)\right)^{2},\left(x_{2 r}^{\alpha}(t)\right)^{2}\right]+\int_{0}^{t}(t-s)\left[\left(x_{2 l}^{\alpha}(s)\right),\left(x_{2 r}^{\alpha}(s)\right)\right] d s\right) \\
= & {\left[(\alpha+1) t\left(x_{1 l}^{\alpha}(t)\right)^{2}+\int_{0}^{t}(t-s) x_{1 l}^{\alpha}(s) d s,(3-\alpha) t\left(x_{1 r}^{\alpha}(t)\right)^{2}+\int_{0}^{t}(t-s) x_{1 r}^{\alpha}(s) d s\right] } \\
& \times\left[(\alpha+1) t\left(x_{2 l}^{\alpha}(t)\right)^{2}+\int_{0}^{t}(t-s) x_{2 l}^{\alpha}(s) d s,(3-\alpha) t\left(x_{2 r}^{\alpha}(t)\right)^{2}+\int_{0}^{t}(t-s) x_{2 r}^{\alpha}(s) d s\right] .
\end{aligned}
$$

Further, we have

$$
\begin{aligned}
& d_{L}\left(\left[f\left(t, x(t), \int_{0}^{t} q(t, s, x(s)) d s\right)\right]^{\alpha},\left[f\left(t, y(t), \int_{0}^{t} q(t, s, y(s)) d s\right)\right]^{\alpha}\right) \\
& =d_{L}\left(\left[(\alpha+1) t\left(x_{i l}^{\alpha}(t)\right)^{2}+\int_{0}^{t}(t-s) x_{i l}^{\alpha}(s) d s,(3-\alpha) t\left(x_{i r}^{\alpha}(t)\right)^{2}+\int_{0}^{t}(t-s) x_{i r}^{\alpha}(s) d s\right],\right. \\
& \left.\quad\left[(\alpha+1) t\left(y_{i l}^{\alpha}(t)\right)^{2}+\int_{0}^{t}(t-s) y_{i l}^{\alpha}(s) d s,(3-\alpha) t\left(y_{i r}^{\alpha}(t)\right)^{2}+\int_{0}^{t}(t-s) y_{i r}^{\alpha}(s) d s\right]\right) \\
& =t \max _{1 \leq i \leq 2}\left\{(\alpha+1)\left|\left(x_{i l}^{\alpha}(t)\right)^{2}-\left(y_{i l}^{\alpha}(t)\right)^{2}\right|,(3-\alpha)\left|\left(x_{i r}^{\alpha}(t)\right)^{2}-\left(y_{i r}^{\alpha}(t)\right)^{2}\right|\right\} \\
& +\int_{0}^{t}(t-s) \max _{1 \leq i \leq 2}\left\{\left|x_{i l}^{\alpha}(s)-y_{i l}^{\alpha}(s)\right|,\left|x_{i r}^{\alpha}(s)-y_{i r}^{\alpha}(s)\right|\right\} d s
\end{aligned}
$$




$$
\begin{aligned}
\leq & T(3-\alpha) \max _{1 \leq i \leq 2}\left\{\left|x_{i l}^{\alpha}(t)-y_{i l}^{\alpha}(t)\right|\left|x_{i l}^{\alpha}(t)+y_{i l}^{\alpha}(t)\right|,\left|x_{i r}^{\alpha}(t)-y_{i r}^{\alpha}(t)\right|\left|x_{i r}^{\alpha}(t)+y_{i r}^{\alpha}(t)\right|\right\} \\
& +\frac{T^{2}}{2} \max _{1 \leq i \leq 2}\left\{\left|x_{i l}^{\alpha}(t)-y_{i l}^{\alpha}(t)\right|,\left|x_{i r}^{\alpha}(t)-y_{i r}^{\alpha}(t)\right|\right\} \\
\leq & 3 T\left|x_{i r}^{\alpha}(t)+y_{i r}^{\alpha}(t)\right| d_{L}\left([x(t)]^{\alpha},[y(t)]^{\alpha}\right)+\frac{T^{2}}{2} d_{L}\left([x(t)]^{\alpha},[y(t)]^{\alpha}\right) \\
= & k_{1} d_{L}\left([x(t)]^{\alpha},[y(t)]^{\alpha}\right)+k_{2} d_{L}\left([x(t)]^{\alpha},[y(t)]^{\alpha}\right), \\
d_{L}( & {\left.[g(x(\cdot))]^{\alpha},[g(y(\cdot))]^{\alpha}\right)=d_{L}\left(\left[\sum_{k=1}^{p} c_{k}\left(x\left(t_{k}\right)\right)\right]^{\alpha},\left[\sum_{k=1}^{p} c_{k}\left(y\left(t_{k}\right)\right)\right]^{\alpha}\right) } \\
= & \left.\max _{1 \leq i \leq 2}\left\{\left|\sum_{k=1}^{p}\left(c_{k}\right)_{i}\left(x_{i l}^{\alpha}\left(t_{k}\right)\right)-\sum_{k=1}^{p}\left(c_{k}\right)_{i}\left(y_{i l}^{\alpha}\left(t_{k}\right)\right)\right|, \mid \sum_{k=1}^{p}\left(c_{k}\right)\right)_{i}\left(x_{i r}^{\alpha}\left(t_{k}\right)\right)-\sum_{k=1}^{p}\left(c_{k}\right)_{i}\left(y_{i r}^{\alpha}\left(t_{k}\right)\right) \mid\right\} \\
\leq & \left|\sum_{k=1}^{p} c_{k}\right| \max _{1 \leq i \leq 2}\left\{\left|x_{i l}^{\alpha}\left(t_{k}\right)-y_{i l}^{\alpha}\left(t_{k}\right)\right|,\left|x_{i r}^{\alpha}\left(t_{k}\right)-y_{i r}^{\alpha}\left(t_{k}\right)\right|\right\}=\left|\sum_{k=1}^{p} c_{k}\right| d_{L}\left(\left[x\left(t_{k}\right)\right]^{\alpha},\left[y\left(t_{k}\right)\right]^{\alpha}\right) \\
\leq & \left|\sum_{k=1}^{p} c_{k}\right| \max _{k} d_{L}\left(\left[x\left(t_{k}\right)\right]^{\alpha},\left[y\left(t_{k}\right)\right]^{\alpha}\right)=h d_{L}\left([x(\cdot)]^{\alpha},[y(\cdot)]^{\alpha}\right),
\end{aligned}
$$

where $k_{1}, k_{2}$, and $h$ satisfy inequalities (3.7), (3.8), and (3.9), respectively. Choose $T$ such that $T<(1-c(h+d)) / c$. Then all conditions stated in Theorem 3.3 are satisfied, so the problem (5.7) has a unique fuzzy solution.

Let target set be $x^{1}=\left(x_{1}^{1}, x_{2}^{1}\right)=(\widetilde{2}, \widetilde{3})$. The $\alpha$-level set of fuzzy numbersis $\widetilde{3}[\widetilde{3}]^{\alpha}=$ $[\alpha+2,4-\alpha]$.

From the definition of fuzzy solution,

$$
\begin{aligned}
x_{i l}^{\alpha}(t)= & S_{i l}^{\alpha}(t)\left(\left(x_{0}\right)_{i l}^{\alpha}-\sum_{k=1}^{p}\left(c_{k}\right)_{i}\left(x_{i l}^{\alpha}\left(t_{k}\right)\right)\right) \\
& +\int_{0}^{t} S_{i l}^{\alpha}(t-s)\left((\alpha+1) s\left(x_{i l}^{\alpha}(s)\right)^{2}+\int_{0}^{s}(s-\tau) x_{i l}^{\alpha} d \tau\right) d s \\
& +\int_{0}^{t} S_{i l}^{\alpha}(t-s) u_{i l}^{\alpha}(s) d s+\sum_{0<t_{k}<t} S_{i l}^{\alpha}\left(t-t_{k}\right) I_{k i l}^{\alpha}\left(x\left(t_{k}^{-}\right)\right), \\
x_{i r}^{\alpha}(t)= & S_{i r}^{\alpha}(t)\left(\left(x_{0}\right)_{i r}^{\alpha}-\sum_{k=1}^{p}\left(c_{k}\right)_{i}\left(x_{i r}^{\alpha}\left(t_{k}\right)\right)\right) \\
& +\int_{0}^{t} S_{i r}^{\alpha}(t-s)\left((3-\alpha) s\left(x_{i r}^{\alpha}(s)\right)^{2}+\int_{0}^{s} x_{i r}^{\alpha} d \tau\right) d s \\
& +\int_{0}^{t} S_{i r}^{\alpha}(t-s) u_{i r}^{\alpha}(s) d s+\sum_{0<t_{k}<t} S_{i r}^{\alpha}\left(t-t_{k}\right) I_{k i r}^{\alpha}\left(x\left(t_{k}^{-}\right)\right),
\end{aligned}
$$

where $i=1,2$. 
Thus the $\alpha$-levels of $u(s)$

$$
\begin{aligned}
& u_{1 l}^{\alpha}(s)=\left(\tilde{\beta}_{1 l}^{\alpha}\right)^{-1}\left((\alpha+1)-\sum_{k=1}^{p}\left(c_{k}\right)_{1}\left(x_{i l}^{\alpha}\left(t_{k}\right)\right)\right. \\
& -\left[S_{1 l}^{\alpha}(T)\left((\alpha-1)-\sum_{k=1}^{p}\left(c_{k}\right)_{1}\left(x_{1 l}^{\alpha}\left(t_{k}\right)\right)\right)\right. \\
& \left.-\int_{0}^{T}(\alpha+1) S_{1 l}^{\alpha}(T-s)\left(s\left(x_{1 l}^{\alpha}(s)\right)^{2}+\int_{0}^{s}(s-\tau) x_{1 l}^{\alpha}(\tau) d \tau\right) d s\right] \\
& \left.-\sum_{0<t_{k}<T} S_{1 l}^{\alpha}\left(T-t_{k}\right) I_{k 1 l}^{\alpha}\left(x_{1 l}^{\alpha}\left(t_{k}^{-}\right)\right)\right), \\
& u_{1 r}^{\alpha}(s)=\left(\tilde{\beta}_{1 r}^{\alpha}\right)^{-1}\left((3-\alpha)-\sum_{k=1}^{p}\left(c_{k}\right)_{1}\left(x_{i r}^{\alpha}\left(t_{k}\right)\right)\right. \\
& -\left[S_{1 r}^{\alpha}(T)\left((1-\alpha)-\sum_{k=1}^{p}\left(c_{k}\right)_{1}\left(x_{1 r}^{\alpha}\left(t_{k}\right)\right)\right)\right. \\
& \left.-\int_{0}^{T}(3-\alpha) S_{1 r}^{\alpha}(T-s)\left(s\left(x_{1 r}^{\alpha}(s)\right)^{2}+\int_{0}^{s}(s-\tau) x_{1 r}^{\alpha}(\tau) d \tau\right) d s\right] \\
& \left.-\sum_{0<t_{k}<T} S_{1 r}^{\alpha}\left(T-t_{k}\right) I_{k 1 r}^{\alpha}\left(x_{1 r}^{\alpha}\left(t_{k}^{-}\right)\right)\right) \\
& u_{2 l}^{\alpha}(s)=\left(\tilde{\beta}_{2 l}^{\alpha}\right)^{-1}\left((\alpha+2)-\sum_{k=1}^{p}\left(c_{k}\right)_{2}\left(x_{i l}^{\alpha}\left(t_{k}\right)\right)\right. \\
& -\left[S_{2 l}^{\alpha}(T)\left((\alpha-1)-\sum_{k=1}^{p}\left(c_{k}\right)_{2}\left(x_{2 l}^{\alpha}\left(t_{k}\right)\right)\right)\right. \\
& \left.-\int_{0}^{T}(\alpha+1) S_{2 l}^{\alpha}(T-s)\left(s\left(x_{2 l}^{\alpha} s\right)^{2}+\int_{0}^{s}(s-\tau) x_{2 l}^{\alpha}(\tau) d \tau\right) d s\right] \\
& \left.-\sum_{0<t_{k}<T} S_{2 l}^{\alpha}\left(T-t_{k}\right) I_{k 2 l}^{\alpha}\left(x_{2 l}^{\alpha}\left(t_{k}^{-}\right)\right)\right), \\
& u_{2 r}^{\alpha}(s)=\left(\tilde{\beta}_{2 r}^{\alpha}\right)^{-1}\left((4-\alpha)-\sum_{k=1}^{p}\left(c_{k}\right)_{2}\left(x_{i r}^{\alpha}\left(t_{k}\right)\right)\right. \\
& \text { - }\left[S_{2 r}^{\alpha}(T)\left((1-\alpha)-\sum_{k=1}^{p}\left(c_{k}\right)_{2}\left(x_{2 r}^{\alpha}\left(t_{k}\right)\right)\right)\right. \\
& \left.-\int_{0}^{T}(3-\alpha) S_{2 r}^{\alpha}(T-s)\left(s\left(x_{2 r}^{\alpha}(s)\right)^{2}+\int_{0}^{s}(s-\tau) x_{2 r}^{\alpha}(\tau) d \tau\right) d s\right] \\
& \left.-\sum_{0<t_{k}<T} S_{2 r}^{\alpha}\left(T-t_{k}\right) I_{k 2 r}^{\alpha}\left(x_{2 r}^{\alpha}\left(t_{k}^{-}\right)\right)\right) \text {. }
\end{aligned}
$$


Then $\alpha$-level of $x(T)=\left(x_{1}(T), x_{2}(T)\right)$ is

$$
\begin{aligned}
& {\left[x_{1}(T)\right]^{\alpha}=\left[x_{1 l}^{\alpha}(T), x_{1 r}^{\alpha}(T)\right]} \\
& =\left[S_{1 l}^{\alpha}(T)\left((\alpha-1)-\sum_{k=1}^{p}\left(c_{k}\right)_{1}\left(x_{1 l}^{\alpha}\left(t_{k}\right)\right)\right)\right. \\
& +\int_{0}^{T}(\alpha+1) S_{1 l}^{\alpha}(T-s)\left(s\left(x_{1 l}^{\alpha}(s)\right)^{2}+\int_{0}^{s}(s-\tau) x_{1 l}^{\alpha}(\tau) d \tau\right) d s \\
& +\tilde{\beta}_{1 l}^{\alpha}\left(\tilde{\beta}_{1 l}^{\alpha}\right)^{-1}\left((\alpha+1)-\sum_{k=1}^{p}\left(c_{k}\right)_{1}\left(x_{i l}^{\alpha}\left(t_{k}\right)\right)\right. \\
& -\left\{S_{1 l}^{\alpha}(T)\left((\alpha-1)-\sum_{k=1}^{p}\left(c_{k}\right)_{1}\left(x_{1 l}^{\alpha}\left(t_{k}\right)\right)\right)\right. \\
& +\int_{0}^{T}(\alpha+1) S_{1 l}^{\alpha}(T-s)\left(s\left(x_{1 l}^{\alpha}(s)\right)^{2}+\int_{0}^{s}(s-\tau) x_{1 l}^{\alpha}(\tau) d \tau\right) d s \\
& \left.\left.+\sum_{0<t_{k}<T} S_{1 l}^{\alpha}\left(T-t_{k}\right) I_{k 1 l}^{\alpha}\left(x_{1 l}^{\alpha}\left(t_{k}^{-}\right)\right)\right\}\right) \\
& +\sum_{0<t_{k}<T} S_{1 l}^{\alpha}\left(T-t_{k}\right) I_{k 1 l}^{\alpha}\left(x_{1 l}^{\alpha}\left(t_{k}^{-}\right)\right), S_{1 r}^{\alpha}(T)\left((1-\alpha)-\sum_{k=1}^{p}\left(c_{k}\right)_{1}\left(x_{1 r}^{\alpha}\left(t_{k}\right)\right)\right) \\
& +\int_{0}^{T}(3-\alpha) S_{1 r}^{\alpha}(T-s)\left(s\left(x_{1 r}^{\alpha}(s)\right)^{2}+\int_{0}^{s}(s-\tau) x_{1 r}^{\alpha}(\tau) d \tau\right) d s \\
& +\tilde{\beta}_{1 r}^{\alpha}\left(\tilde{\beta}_{1 r}^{\alpha}\right)^{-1}\left((3-\alpha)-\sum_{k=1}^{p}\left(c_{k}\right)_{1}\left(x_{1 r}^{\alpha}\left(t_{k}\right)\right)\right. \\
& -\left\{S_{1 r}^{\alpha}(T)\left((1-\alpha)-\sum_{k=1}^{p}\left(c_{k}\right)_{1}\left(x_{1 r}^{\alpha}\left(t_{k}\right)\right)\right)\right. \\
& +\int_{0}^{T}(3-\alpha) S_{1 r}^{\alpha}(T-s)\left(s\left(x_{1 r}^{\alpha}(s)\right)^{2}+\int_{0}^{s}(s-\tau) x_{1 r}^{\alpha}(\tau) d \tau\right) d s \\
& \left.\left.+\sum_{0<t_{k}<T} S_{1 r}^{\alpha}\left(T-t_{k}\right) I_{k 1 r}^{\alpha}\left(x_{1 r}^{\alpha}\left(t_{k}^{-}\right)\right)\right\}\right) \\
& \left.+\sum_{0<t_{k}<T} S_{1 r}^{\alpha}\left(T-t_{k}\right) I_{k 1 r}^{\alpha}\left(x_{1 r}^{\alpha}\left(t_{k}^{-}\right)\right)\right] \\
& =\left[(\alpha+1)-\sum_{k=1}^{p}\left(c_{k}\right)_{1}\left(x_{1 l}^{\alpha}\left(t_{k}\right)\right),(3-\alpha)-\sum_{k=1}^{p}\left(c_{k}\right)_{1}\left(x_{1 r}^{\alpha}\left(t_{k}\right)\right)\right] \\
& =\left[\tilde{2}-\sum_{k=1}^{p}\left(c_{k}\right)_{1}\left(x_{1}\left(t_{k}\right)\right)\right]^{\alpha} \text {. }
\end{aligned}
$$


Similarly,

$$
\left[x_{2}(T)\right]^{\alpha}=\left[x_{2 l}^{\alpha}(T), x_{2 r}^{\alpha}(T)\right]=\left[\widetilde{3}-\sum_{k=1}^{p}\left(c_{k}\right)_{2}\left(x_{2}\left(t_{k}\right)\right)\right]^{\alpha} .
$$

Hence

$$
x(T)=\left(x_{1}(T), x_{2}(T)\right)=\left(\tilde{2}-\sum_{k=1}^{p}\left(c_{k}\right)_{1}\left(x_{1}\left(t_{k}\right)\right), \tilde{3}-\sum_{k=1}^{p}\left(c_{k}\right)_{2}\left(x_{2}\left(t_{k}\right)\right)\right)=x^{1}-g(x) .
$$

Then all the conditions stated in Theorem 4.2 are satisfied, so the system (5.7) is nonlocal controllable on $[0, T]$.

\section{Acknowledgment}

This study was supported by research funds from Dong-A University.

\section{References}

[1] D. D. Bainov and P. S. Simenov, Impulsive Differential Equations: Periodic Solutions and Applications, Longman, Harlow, UK, 1993.

[2] V. Lakshmikantham, D. D. BaĬnov, and P. S. Simeonov, Theory of Impulsive Differential Equations, vol. 6 of Series in Modern Applied Mathematics, World Scientific, Singapore, 1989.

[3] A. M. Samoileuko and N. A. Perestyuk, Impulsive Differential Equations, vol. 28 of Series on Advances in Mathematics for Applied Sciences, World Scientific, Singapore, 1995.

[4] Y. V. Rogovchenko, "Impulsive evolution systems: main results and new trends," Dynamics of Continuous, Discrete and Impulsive Systems, vol. 3, no. 1, pp. 57-88, 1997.

[5] V. Lakshmikantham and F. A. McRae, "Basic results for fuzzy impulsive differential equations," Mathematical Inequalities E Applications, vol. 4, no. 2, pp. 239-246, 2001.

[6] J. H. Park, J. S. Park, Y. C. Ahn, and Y. C. Kwun, "Controllability for the impulsive semilinear fuzzy integrodifferential equations," Advances in Soft Computing, vol. 40, pp. 704-713, 2007.

[7] R. Rodríguez-López, "Periodic boundary value problems for impulsive fuzzy differential equations," Fuzzy Sets and Systems, vol. 159, no. 11, pp. 1384-1409, 2008.

[8] P. Balasubramaniam and S. Muralisankar, "Existence and uniqueness of fuzzy solution for semilinear fuzzy integrodifferential equations with nonlocal conditions," Computers $\mathcal{E}$ Mathematics with Applications, vol. 47, no. 6-7, pp. 1115-1122, 2004.

[9] J. H. Park, J. S. Park, and Y. C. Kwun, "Controllability for the semilinear fuzzy integrodifferential equations with nonlocal conditions," in Proceedings of the 3rd International Conference on Fuzzy Systems and Knowledge Discovery (FSKD '06), vol. 4223 of Lecture Notes in Artificial Intelligence, pp. 221-230, 2006.

[10] Y. C. Kwun, M. J. Kim, B. Y. Lee, and J. H. Park, “Existence of solutions for the semilinear fuzzy integrodifferential equations using by succesive iteration," Journal of Korean Institute of Intelligent Systems, vol. 18, pp. 543-548, 2008.

[11] Y. C. Kwun, M. J. Kim, J. S. Park, and J. H. Park, "Continuously initial observability for the semilinear fuzzy integrodifferential equations," in Proceedings of the 5th International Conference on Fuzzy Systems and Knowledge Discovery (FSKD '08), vol. 1, pp. 225-229, 2008.

[12] B. Bede and S. G. Gal, "Almost periodic fuzzy-number-valued functions," Fuzzy Sets and Systems, vol. 147, no. 3, pp. 385-403, 2004. 
[13] S. G. Gal and G. M. N'Guerekata, "Almost automorphic fuzzy-number-valued functions," Journal of Fuzzy Mathematics, vol. 13, no. 1, pp. 185-208, 2005.

[14] Y. C. Kwun, J. S. Kim, M. J. Park, and J. H. Park, “Nonlocal controllability for the semilinear fuzzy integrodifferential equations in $n$-dimensional fuzzy vector space," Advances in Difference Equations, vol. 2009, Article ID 734090, 16 pages, 2009.

[15] G. Wang, Y. Li, and C. Wen, “On fuzzy n-cell numbers and n-dimension fuzzy vectors," Fuzzy Sets and Systems, vol. 158, no. 1, pp. 71-84, 2007. 